\title{
Erratum: A Monte Carlo Study of the Rotation-Libration Transition in Solid $\mathrm{H}_{2}$ Under Pressure*
}

\author{
I. Aviram and S. Goshen \\ Nuclear Research Center Negev, Beer-Sheva, Israel \\ and $\mathbf{R}$. Thieberger \\ Nuclear Research Center Negev, Beer-Sheva, Israel and Department of Physics, Ben Gurion \\ University of the Negev, Beer-Sheva, Israel \\ (Received August 18, 1980) \\ In Eq. (9) the number $10^{7}$ should be replaced by $10^{6}$. The results \\ presented in the paper are based on the correct value.
}

*This paper appeared in J. Low Temp. Phys. 38, 371 (1980). 